A comparison with these two examples and with many other cases gives an indication of the size and direction of the error in the computation. The most probable value for the ${ }^{5} \mathrm{~S}$ state of carbon is close to $98 \mathrm{kcal} . / \mathrm{mole}$, in good agreement with Edlén's estimate of $94 \pm 2 \mathrm{kcal} . / \mathrm{mole}$.

Department of Physics,

S. Goudsmit

Northwestern University,

Evanston, Illinois.

March 10.

${ }^{1}$ Nature, 159, 129 (1947). ${ }^{2}$ Bacher, R. F., and Goudsmit, S., Phys. Rev., 46, 948 (1934), see p.

\section{Electronic Function Generator}

IT is interesting to compare the accompanying diagram with that given by Mr. D. M. MacKay in a recent communication ${ }^{1}$. The present diagram is from the original sketch used in a paper I read to the Rugby Engineering Society on February 6, $1946^{2}$. This particular embodiment of the idea was demonstrated on the same occasion.

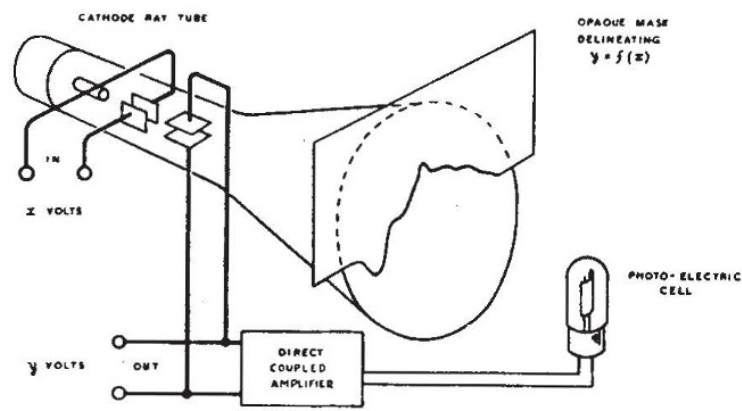

In addition to the applications mentioned by $\mathrm{Mr}$. MacKay, it is possible to combine a pair of electronic function generators to form a high-speed electronic analogue multiplying unit. This aspect has been dealt with in an article which was accepted for publication ${ }^{3}$ towards the end of 1946.

A provisional patent application was filed by me and the British Thomson-Houston Co., Ltd., early in $1946^{4}$, and pre-dates the application quoted by Mr. MacKay by some ten months. A complete specification was filed on January 14 this year. D. J. MYNALI

Electronics Engineering Department, British Thomson-Houston Co., Ltd., Rugby.

${ }^{1}$ Nature, 159, 406 (1947).

2 "Elementary Calculations by Electrical Means".

${ }^{3}$ Electronic Engineering, in the press.

4 Brit. Prov. Pat. Spec. No. 3285/46.

\section{Constitutions of Dinitrogen Tetroxide and Trioxide}

THE structure of dinitrogen tetroxide has recently been much discussed, but no clear conclusion has been reached. The structure of dinitrogen trioxide has less often been explicitly considered, but it, too, has remained uncertain.

In general terms, the position with regard to dinitrogen tetroxide has been that, while physical evidence has pointed to structure I, chemical evidence has been held to require some structure, such as II, which is capable of dividing, in addition reactions, to produce a nitroso-group inter alia. Structure III has been suggested, but not seriously supported. One proposal for satisfying both the physical and chemical requirements has been to assume forms such as I, II and III in equilibrium ${ }^{1}$. Structure IV represents a very special concept which has been introduced ${ }^{2}$ in another attempt to reconcile the physical and chemical indications. (For simplicity, mesomeric states will be represented in each case by a principal valency structure.)

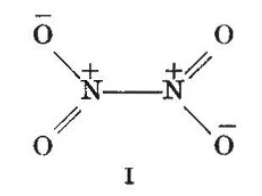

$\mathrm{O}=\mathrm{N}-\mathrm{O}-\mathrm{O}-\mathrm{N}=\mathrm{O}$

III<smiles>O=NO[N+](=O)O</smiles>

o<smiles>O=[N+]1O[NH+]([O-])O1</smiles>

IV
The main point of the chemical evidence has been that, according to the older organic chemical literature, a variety of groups, including nitroso-, nitro-, nitrito- and nitrato-groups, have been introduced into organic compounds by the action of dinitrogen tetroxide ${ }^{3}$. However, this evidence is largely annulled by the recent work of Levy and Scaife ${ }^{4}$, from which it is clear that the complicated picture presented by the older literature is the result of an insufficiently exact control of experimental conditions. These authors show that there are but two primary products of the addition of dinitrogen tetroxide to simple olefins, namely, dinitro-compounds and nitro-nitrito-compounds, and that, in the latter products, the nitrogroup attaches itself to that carbon atom which carries the larger number of hydrogen atoms. They conclude that, so far as their results are concerned, there is little to choose between formulæ I and II; but we are going to suggest that, considered in a somewhat wider setting, their work completes a chain of evidence leading to a more specific inference.

As regards physical evidence on the structure of dinitrogen tetroxide, some firm conclusions can be drawn from the spectroscopic work of Sutherland ${ }^{5}$. This shows that the molecule possesses a centre of symmetry, and that it has a low, very intense, Raman frequency, such as would arise from a weak central bond. It also excludes the presence, in any large amount, of any tautomer not fulfilling these conditions. This indicates formula $I$, though it does not clearly exclude either III or IV. (Mesomerism confers an NN-link on IV.) Formula $I$ is also supported by Giauque and Kemp's measurements of the entropy of dinitrogen tetroxide ${ }^{6}$; and Hendricks has shown ${ }^{2}$ that the same structure provides a consistent interpretation of Végard's X-ray data ${ }^{8}$.

The additive reactivity of dinitrogen tetroxide shows that it is an electrophilic addendum, and adds in the successive parts nitronium ion and nitrite ion. The nitronium ion goes where a proton would in an addition of hydrogen chloride, and it necessarily forms a nitro-group, while the nitrite ion can attach itself to the remaining position either as a nitro- or as a nitrito-group. Either formula I or II can fulfil these conditions, and using formula I the necessary polarization is illustrated in V. We need not consider the question of the free life, if any, of the anionic component of the addendum, since this must depend on 